\title{
¿Podemos favorecer el proceso de aprendizaje y la inclusión social y académica en la universidad de los estudiantes con más dificultades? Propuesta de un estudio de investigación
}

\author{
Chust Hernández,Pablo \\ ServiciodeOrientaciónAcadémica,UniversidadCatólicadeValencia,España \\ pablo.chust@ucv.es \\ Senent Sánchez, JoanMaría \\ Facultat de Filosofía y Ciencias de la Educación, Universitat de València(España) \\ senent@uv.es
}

\section{Resumen}

Durante el primer curso universitario, los estudiantes deben hacer frente a nuevas exigencias académicas, (evaluación, metodologías docentes...), con el fin de superar ese primer curso, al tiempo de integrarse socialmente en un contexto, la universidad, nuevo para ellos. Para ello, deben adquirir o reforzar distintas habilidades. La literatura científica ha evidenciado la relación entre estas variables con el rendimiento académico y el ajuste al primer año de universidad: actitudes ante el aprendizaje (Gargallo et al, 2007-A-), estrategias de aprendizaje (Gargallo et al., 2009), estrés académico (Rayle y Chung, 2007; Robotham y Julian 2006), autoeficacia académica (Palacio et al., 2012; Salanova et al., 2005), ansiedad ante los exámenes (Lang y Lang, 2010; Salanova et al., 2005) e integración social en el grupo/clase (Álvarez et al., 2011; Rausch y Hamilton, 2006).

Para que las universidades puedan garantizar una formación eficaz y de calidad es necesario analizar aspectos vinculados al éxito académico (Belvis et al, 2009) y llevar a cabo acciones que permitan perfeccionar los procesos de enseñanza-aprendizaje.

En esta línea, se propone la realización de un estudio longitudinal, cuyo objetivo es analizar la evolución, durante el $1^{\circ}$ curso universitario, de variables que inciden en el rendimiento académico del alumnado, medidas mediante cuestionarios validados para población universitaria española:

- Actitudes ante el aprendizaje

- Estrategias de aprendizaje

- Autoeficacia académica

- Estrés académico

- Ansiedad ante los exámenes

- Integración social

La población diana serán todos los estudiantes de nuevo ingreso en grados de la Universidad Católica de Valencia (curso 2017-2018). Se les aplicaron los cuestionarios al inicio del $1^{\circ}$ curso y se aplicarán de nuevo a principios de $2^{\circ}$ curso. Los resultados mostrarán las habilidades que no evolucionan adecuadamente durante este primer curso. Ello posibilitará la implementación de acciones dirigidas a perfeccionarlas, optimizando así los procesos de enseñanza-aprendizaje de los estudiantes.

\section{Abstract}

During the first university year, students must face new academic demands (assessment, teaching methodologies, etc.) in order to pass the first year, at the same time as integrating socially into a new context, the university, which is entirely unfamiliar to them. Therefore, they must acquire or reinforce different skills. The scientific literature has shown the relationship between these variables with academic performance and first-year college adjustment: Attitudes to learning (Gargallo et al, 2007), learning strategies (Gargallo et al, 2009), academic stress (Rayle and Chung, 2007; Robotham and Julian 2006), academic self-efficacy (Palacio et al, 2012; Salanova et al, 2005), anxiety about exams (Lang and Lang, 2010; Salanova et al, 2005) and social integration in the group/class (Álvarez et al, 2011; Raush and Hamilton, 2006).

To guarantee effective and quality training, universities need to analyse aspects related to academic success (Belvis et al, 2009) and carry out actions to optimize teaching-learning processes. 
In this sense, a longitudinal study is proposed, with the objective of analysing the evolution, during the first university year, of variables that affect the academic performance of students, measured by means of questionnaires validated for the Spanish university population:

- Attitudes towards learning

- Learning strategies

- Academic self-efficacy

- Academic stress

- Anxiety about exams

- Social integration

The target population will include all incoming students in the different faculties of the Catholic University of Valencia (academic year 2017-2018). The questionnaires are applied to the students at the beginning of the first year and will be applied again at the beginning of the second year.The results will highlight skills that do not evolve properly during this first course,enabling the implementation of actions aimed at improving them, thus optimizing the teaching-learning processes of students.

Palabras clave: Estrategias de aprendizaje, Actitudes ante el aprendizaje, Estrés académico, Autoeficacia Académica, Ansiedad ante los exámenes, Estudiantes Universitarios, Educación Superior, Inclusión e integración social y académica.

Keywords: Learning strategies, Learning attitudes, Academic stress, Academic self-efficacy, Test anxiety, University Students, Higher Education, Social and academic inclusion and integration.

\section{INTRODUCCIÓN}

El primer curso universitario supone para los alumnos un cambio que requiere una adaptación a nivel social, personal y académico (Pulido et al, 2011). Con respecto a lo académico, aunque casi todo el alumnado tiene experiencia previa en otros niveles de estudio (Bachiller, Formación Profesional, etc.), lo cierto es que los universitarios de nuevo ingreso tienen que hacer frente a una serie de demandas nuevas y diferentes a las que hasta ahora se han enfrentado (diferentes formas de evaluación, metodologías docentes nuevas para él...).

Para poder hacer frente a estas exigencias y poder superar con éxito el primer curso de universidad, alcanzando un adecuado rendimiento académico, los estudiantes deben adquirir una serie de habilidades.

Las variables que inciden en el rendimiento académico de los estudiantes universitarios son múltiples y complejas, y constituyen una maraña en la que se hace muy difícil ponderar la influencia concreta de cada una (Gargallo et al. 2007-A-). Sin embargo, analizando la literatura científica existente, podemos observar como hay una serie de variables de corte académico, que influyen en el rendimiento, y que integran una serie de habilidades y actitudes que son susceptibles de ser enseñadas a los alumnos, con el objetivo de que puedan controlarlas, mejorando así su proceso de aprendizaje.

Así, en las últimas dos décadas se han estudiado las relaciones entre algunas de estas variables con el rendimiento académico y el ajuste al primer año de universidad: actitudes ante el aprendizaje (Gargallo et al., 2007-A-), estrategias de aprendizaje (Juárez et al., 2016; Bahamón et al., 2013; Yip, 2012; Gargallo et al., 2009; Gargallo et al., 2007-B-), estrés académico (Hamaideh et al., 2016; Rayle y Chung, 2007; Robotham y Julian 2006; Lépine et al., 2004), ansiedad ante los exámenes (Khalaila, 2015; Bonaccio et al, 2012; Lang y Lang, 2010; Salanova et al., 2005; Chapell et al., 2005), autoeficacia académica (Palacio et al., 2012; Salanova et al., 2005) e integración social en el grupo/clase (Álvarez et al., 2011; Rausch y Hamilton, 2006).

Para que las universidades puedan garantizar una formación de calidad, en la que los estudiantes puedan lograr un óptimo rendimiento académico, se hace necesario analizar aspectos vinculados al éxito académico (Belvis et al., 2009) con el objetivo de llevar a cabo medidas y acciones que permitan perfeccionar los procesos deenseñanza-aprendizaje. 


\section{JUSTIFICACIÓN DEL ESTUDIO}

Aunque la dinámica del primer curso universitario y el mismo trabajo que conlleva ya proporciona al alumnado la adquisición de parte de las habilidades comentadas en el apartado anterior, para la consecución de otras será necesario un refuerzo/apoyo por parte de equipos de orientación académica o del personal docente; así y todo, es posible que otra parte de dichas habilidades no mejoren con dichos refuerzos durante el $1^{\circ}$ curso en todos los alumnos, y que sea necesario un apoyo extra para que los estudiantes las adquieran al inicio del $2^{\circ}$ curso.

El ranking de universidades, realizado en 2017 por la Fundación BBVA y por el Instituto Valenciano de Investigaciones Económicas -IVIE- (Fundación BBVA e Instituto Valenciano de Investigaciones Económicas, 2017) evalúa, entre otros indicadores, los siguientes:

- Tasa de Éxito (número de créditos aprobados -excluyendo adaptados, convalidados y reconocidos- respecto al total de créditosevaluados)

- Tasa de Evaluación (número de créditos evaluados respecto al total de créditos matriculados)

- Tasa de Abandono (alumnos que, dos años después de haberse matriculado en el primer curso de una titulación, la abandonan singraduarse)

En dicho ranking, se muestra que la Universidad Católica de Valencia (en adelante UCV) tiene una tasa de éxito ligeramente superior al $70 \%$, una tasa de evaluación ligeramente superior al $80 \%$ y una tasa de abandono del 10\% (http://www.u-ranking.es/descargas/fichas/06-u-ranking-2017-UCV.pdf). Estos resultados revelan que en la UCV, la media de los alumnos suspende, por cada curso, 3 créditos de cada 10 que son evaluados, y que uno de cada 10 alumnos termina abandonando la universidad. Desde nuestra perspectiva, creemos necesario concentrar esfuerzos por reducir estas tasas lo máximo posible, desde los diferentes servicios de apoyo al estudiante, así como mediante la acción del personal docente.

De dichos créditos suspendidos, una parte se suspende en $1^{\circ}$ curso, pero otra en $2^{\circ}$ y cursos posteriores, lo que muestra que muchos alumnos pasan al $2^{\circ}$ curso de universidad sin haber adquirido las habilidades académicas necesarias que permitan optimizar su rendimiento académico.

Conocer las variables que evolucionan favorablemente y aquellas que no experimentan mejoría durante el $1^{\circ}$ curso, puede ayudar, a los diferentes servicios y equipos que trabajan con los alumnos de la UCV (Servicio de Orientación, Discapacidad, tutores...), así como al profesorado, a mejorar sus intervenciones de cara a facilitar la adquisición de aquellos conocimientos, habilidades y estrategias relativas a las variables que no evolucionen de forma espontánea durante el $1^{\circ}$ curso académico.

Así pues, se prevé realizar un estudio descriptivo que analice la evolución de dichas variables académicas de $1^{\circ}$ a $2^{\circ}$ curso. El estudio se realiza dentro del marco de la realización de un proyecto premiado en los premios INNOVA-UCV, otorgado por la UCV, el día 25 de enero de 2017, en Valencia.

\section{OBJETIVO}

Analizar la evolución, durante el $1^{\circ}$ curso de universidad, de diferentes variables que inciden en el rendimiento académico de los estudiantes de nuevo ingreso de la UCV, medidas con los diferentes cuestionarios (actitudes ante el aprendizaje, estrategias de aprendizaje, estrés académico, ansiedad ante los exámenes, autoeficacia académica, integración social) en función de diversas variables sociodemográficas (sexo y edad), de acceso (vía de acceso a la universidad) y de satisfacción con la experiencia universitaria (satisfacción con la propia capacidad, con la docencia, con los contenidos docentes y con las propias habilidades comunicativas).

\section{MATERIAL Y MÉTODOS}

\subsection{Diseño del estudio}

Estudio cuantitativo, observacional, longitudinal y prospectivo. 


\subsection{Población y muestra}

\subsubsection{Población de estudio}

Todos los estudiantes de nuevo ingreso en grados de la UCV, en el curso 2017-2018.

\subsubsection{Criterios de inclusión}

- Ser estudiante de $1^{\circ}$ curso de cualquier Grado presencial de la UCV durante el curso 2017-2018.

- Tener una edad mínima de 17 años.

- Ser capaz de leer y escribir en español.

- Aceptar de forma voluntaria la participación en el estudio y firmar el consentimiento informado.

\subsubsection{Criterios de exclusión}

— No estar presente en el aula en los dos momentos de aplicación de los cuestionarios.

\subsection{Variables e instrumentos de medida}

El instrumento que se utilizará para recoger los datos tiene un tiempo estimado de administración de entre 20 y 30 minutos, en función de la idiosincrasia de los participantes. Consiste en un cuadernillo formado por los siguientes cuestionarios, todos ellos en idioma castellano y validados para población universitaria española:

— Variable: Actitudes ante el aprendizaje

Instrumento de medida: Cuestionario de Evaluación de Actitudes ante el Aprendizaje en Estudiantes Universitarios -CEVAPU- (Gargallo et al., 2007-C-).

Instrumento de 11 ítems encaminados hacia la evaluación de las actitudes que tienen los estudiantes hacia el aprendizaje en el ámbito universitario. Utiliza una escala tipo Likert con 5 opciones de respuesta. Tiene 3 subescalas:

- Valoración del aprendizaje profundo, con comprensión, disposición activa...

- Valoración y actitud positiva hacia el trabajo en equipo

- Atribuciones internas

— Variable: Estrategias de aprendizaje

Instrumento de medida: Cuestionario de Evaluación de Estrategias de Aprendizaje en Estudiantes Universitarios -CEVEAPEU- (Gargallo et al., 2009).

Instrumento de 88 ítems que evalúa 25 estrategias de aprendizaje, divididas en 6 subescalas. Utiliza una escala tipo Likert con 5 opciones de respuesta. 
Tabla 1. Estructura del CEVEAPEU.

\begin{tabular}{|c|c|}
\hline SUBESCALA & ESTRATEGIAS \\
\hline \multirow{7}{*}{$\begin{array}{l}\text { Subescala } 1 . \\
\text { Estrategias motivacionales }\end{array}$} & 1. Motivación intrínseca \\
\hline & 2. Motivación extrínseca \\
\hline & 3. Valor de la tarea \\
\hline & 4. Atribuciones internas \\
\hline & 5. Atribuciones externas \\
\hline & 6. Autoeficacia y expectativas \\
\hline & 7. Concepción de la inteligencia como modificable \\
\hline \multirow{2}{*}{$\begin{array}{c}\text { Subescala } 2 \text {. } \\
\text { Componentes afectivos }\end{array}$} & 8. Estado físico y anímico \\
\hline & 9. Ansiedad \\
\hline \multirow{4}{*}{$\begin{array}{l}\text { Subescala } 3 \text {. } \\
\text { Estrategias metacognitivas }\end{array}$} & 10. Conocimiento de objetivos y criterios de evaluación \\
\hline & 11. Planificación \\
\hline & 12. Autoevaluación \\
\hline & 13. Control, autorregulación \\
\hline \multirow{2}{*}{$\begin{array}{c}\text { Subescala } 4 . \\
\text { Estrategias de control del contexto, } \\
\text { interacción social y manejo de recursos }\end{array}$} & 14. Control del contexto \\
\hline & 15. Habilidades de interacción social y aprendizaje con compañeros \\
\hline \multirow{2}{*}{$\begin{array}{l}\text { Subescala } 5 . \\
\text { Estrategias de búsqueda y selección de información }\end{array}$} & 16. Conocimiento de fuentes y búsqueda de información \\
\hline & 17. Selección de información \\
\hline \multirow{8}{*}{$\begin{array}{l}\text { Subescala } 6 \text {. } \\
\text { Estrategias de procesamiento y } \\
\text { uso de la información }\end{array}$} & 18. Adquisición de información \\
\hline & 19. Elaboración \\
\hline & 20. Organización \\
\hline & 21. Personalización y creatividad, pensamiento crítico \\
\hline & 22. Almacenamiento. Memorización. Uso de recursos mnemotécnicos \\
\hline & 23. Almacenamiento. Simple repetición \\
\hline & 24. Transferencia. Uso de la información \\
\hline & 25. Manejo de recursos para usar la información adquirida \\
\hline
\end{tabular}

— Variable: Estrés Académico

Cuestionario de Estrés Académico en la Universidad -CEAU- (García-Ros et al., 2012).

Es un instrumento de 19 ítems relativos a situaciones potencialmente estresoras en el ámbito universitario, que utiliza una escala de respuesta tipo Likert con 5 opciones. Tiene 4 subescalas:

- Obligaciones académicas

- Expediente y perspectivas de futuro

- Dificultades interpersonales

- Expresión de ideas propias

— Variable: Ansiedad ante los exámenes

Instrumento de medida: Cuestionario de Ansiedad ante los Exámenes -CAEX- (Valero, 1999). El cuestionario permite evaluar exhaustivamente los problemas de ansiedad ante exámenes, en sus distintos componentes comportamentales, en población universitaria. El instrumento consta de 39 items y utiliza una escala tipo Likert con 6 opciones. Tiene 3 subescalas:

- Preocupación

- Respuestas fisiológicas

- Respuestas de evitación 
— Variable: Autoeficacia Académica

Instrumento de medida: Escala de Autoeficacia Académica General (Torre, 2006).

Instrumento de 9 ítems encaminados hacia la evaluación de la autoeficacia académica que tienen los estudiantes en el ámbito universitario. Utiliza una escala tipo Likert con 5 opciones de respuesta.

- Variable: Integración social

Instrumento de medida: creación propia

— Variable: Satisfacción con la experiencia universitaria

Instrumento de medida: Cuestionario de Satisfacción con la Experiencia Universitaria CSEU - (Kember y Leung, 2005; validado en su versión española por González-Peiteado et al., 2016).

Instrumento de 26 ítems que recoge información acerca de la satisfacción del estudiante con diferentes aspectos de la vida universitaria. Utiliza una escala tipo Likert con 5 opciones de respuesta. Tiene 4 subescalas:

- Satisfacción con la propiacapacidad

- Satisfacción con ladocencia

- Satisfacción con los contenidosdocentes

- Satisfacción con las propias habilidadescomunicativas

— Variables sociodemográficas: sexo, edad, titulación, forma de acceso a la universidad y nº de horas de trabajo a la semana

Instrumento de medida: preguntas de creación propia

\subsection{Temporalización}

Tabla 2. Temporalización del proyecto.

\begin{tabular}{|c|c|c|}
\hline FECHA/PERIODO & TAREA & ESTADO \\
\hline Enero - Julio 2017 & Diseño del estudio & Realizado \\
\hline Marzo - Agosto 2017 & $\begin{array}{l}\text { Aprobación por parte de un comité de } \\
\text { Ética de la Investigación }\end{array}$ & $\begin{array}{l}\text { Realizado. Proyecto aprobado por el Comité de } \\
\text { Ética de la Investigación de la Sociedad } \\
\text { Valenciana de Bioética (4 agosto 2017) }\end{array}$ \\
\hline Septiembre 2017 & $\begin{array}{l}1^{a} \text { fase del estudio empírico: } 1^{\circ} \text { pase de } \\
\text { cuestionarios a los estudiantes de nuevo } \\
\text { ingreso en grados de la UCV }\end{array}$ & Realizado. Total cuestionarios recogidos: 1465 \\
\hline Septiembre 2018 & $\begin{array}{l}2^{\mathrm{a}} \text { fase del estudio empírico: } 2^{\circ} \text { pase de } \\
\text { cuestionarios a los estudiantes de } 2^{\circ} \text { curso } \\
\text { en grados de la UCV }\end{array}$ & No realizado \\
\hline A partir de octubre 2018 & Análisis de resultados & No realizado \\
\hline
\end{tabular}




\subsection{Análisis estadístico}

Los análisis a realizar serán los típicos de las ciencias sociales, es decir, análisis descriptivo de las variables, análisis correlacional, comparativo y modelos de regresión múltiple.

Para analizar las diferencias de medias en muestras independientes, se empleará la prueba T de Student o el ANOVA (pruebas paramétricas). En caso de no normalidad de los datos se analizarán estos con $U$ de Mann-Witney o Kruskall Wallis (pruebas no paramétricas).

Para las diferencias de medias en muestras relacionadas se empleará la prueba $T$ de Student para una misma muestra (prueba paramétrica). En caso de no normalidad de los datos se emplará la W de Wilcoxon (prueba no paramétrica).

Para el análisis correlacional se utilizará la r de Pearson (prueba paramétrica). En caso de no normalidad de los datos, se analizarán con rho de Spearman (prueba no paramétrica)

Se prevé la realización de un modelo de regresión múltiple.

El nivel de significación estadística elegido será siempre de p igual o menor de 0,05.

Para la realización de los análisis se utilizará el paquete estadístico SPSS v23.

\subsection{Aspectos éticos}

Este estudio fue aprobado por el Comité de Ética de la Investigación de la Sociedad Valenciana de Bioética, en Agosto de 2017.

El estudio no supondrá ningún perjuicio para los participantes en el mismo, y responde al principio de no maleficencia. El hecho de que un estudiante no desee participar en el estudio no conllevará ningún perjuicio para él, por parte de ningún miembro activo del equipo investigador ni de la Universidad.

Como en este estudio es necesario incluir únicamente a los estudiantes que contesten a los dos pases del cuestionario, es necesario conocer quiénes son dichos estudiantes. Para ello, previamente al pase de cuestionarios se asignará un código a cada alumno. Ese código servirá para que los investigadores puedan identificar al alumno, con el fin de poder incluir únicamente a aquellos estudiantes que realicen los dos pases de cuestionarios. En ningún momento se pedirán datos identificativos a los alumnos, sino que se les facilitará su código en el momento del pase de cuestionarios, de forma que, desde el primer momento en el que cada investigador salga de las aulas con los cuestionarios contestados, estos no puedan ser identificados por nadie externo al estudio. Por tanto, sólo se podrá identificar a los sujetos utilizando códigos de asociación. Estos códigos únicamente serán conocidos por los investigadores del estudio, que son los miembros del Servicio de Orientación de la UCV.

Todo estudiante que participe en el estudio tendrá derecho de acceso, rectificación y cancelación de los datos, en cualquier momento comprendido entre el principio y el fin de la investigación.

Por otra parte, todo participante podrá tener acceso a la información sobre sí mismo recogida en su cuestionario. Los miembros del servicio de orientación académica de la UCV, que son los investigadores del estudio, podrán proporcionar dicha información, previa petición del alumno.

\section{RESULTADOS DESCRIPTIVOS PRELIMINARES}

Hasta el momento de dicha comunicación, se ha realizado la $1^{\mathrm{a}}$ fase del estudio empírico $\left(1^{\mathrm{a}}\right.$ recogida de datos). La muestra quedó conformada por 1.465 estudiantes, de los cuáles 935 eran mujeres $(63,82 \%)$ y 530 hombres $(36,18 \%)$, provenientes de 28 titulaciones: Magisterio de Educación Primaria, Magisterio de Educación Infantil, Psicología, Educación Social, Logopedia. 
Terapia Ocupacional, Podología, Ciencias de la Actividad Física y Deportiva, Fisioterapia, Nutrición, Enfermería, Medicina, Odontología, Administración y dirección de empresas, Gestión Económico-Financiera, Derecho, Criminología, Multimedia y Artes Digitales, Biotecnología, Veterinaria, Ciencias del Mar, Magisterio de Educación Infantil + Educación Primaria, Magisterio de Educación Primaria + Ciencias de la Actividad Física y Deportiva, Educación Social + Magisterio de Educación Primaria, Educación Social + Trabajo Social, Terapia Ocupacional + Fisioterapia, Derecho + Criminología y Podología + Enfermería.

La edad media de la muestra fue de 19,37 años, siendo la mínima edad 17 años y la mayor 45 . En cuanto a la vía de acceso a la universidad, 607 estudiantes $(41,4 \%)$ son provenientes de Bachillerato de Ciencias y Tecnología, 483 (33\%) de Bachillerato de Ciencias Sociales y Humanidades, y $287(19,6 \%)$ de Ciclo formativo superior. El resto $(6 \%)$ accedieron a la universidad mediante otras vías de acceso (Bachillerato de Artes, otra titulación universitaria, prueba de acceso para mayores de 25 y 40 años).

\section{CONCLUSIONES}

El estudio presentado constituye un sencillo pero interesante intento de analizar diversos aspectos vinculados al éxito académico. Los hallazgos que del estudio se deriven pueden ayudar a comprender la evolución de diversas variables relacionadas con el rendimiento académico, lo que puede ser relevante para el personal docente de las universidades, así como para los distintos servicios de apoyo al estudiante (orientación académica, discapacidad...), de cara a implementar medidas y acciones que permitan perfeccionar los procesos de enseñanza- aprendizaje, brindando a los estudiantes ayudas psicopedagógicas y psicológicas sobre los aspectos en los que presenten mayor problemática.

La sencillez de su realización y el acceso libre de todos los cuestionarios que se utilizan permite la fácil replicación del proyecto en otras instituciones universitarias. 


\section{REFERENCIAS BIBLIOGRÁFICAS}

Álvarez, M., Figuera, P., y Torrado, M. (2011). La problemática de la transición bachillerato- universidad en la Universidad de Barcelona. Revista Española de Orientación y Psicopedagogía, 22(1),15-27.

Bahamón, M., Vianchá, M., Alarcón, L., y Bohórquez, C. (2013). Estilos y estrategias de aprendizaje relacionados con el logro académico en estudiantes universitarios. Pensamiento Psicológico, 11(1),115-129.

Belvis, E., Moreno, M. V., y Ferrer, F. (2009). Los factores explicativos del éxito y fracaso académico en las universidades españolas, en los años del cambio hacia la convergencia europea. Revista Española de Educación Comparada, 15,61-92.

Bonaccio, S., Reeve, L. C., y Winford, C. E. (2012). Test anxiety on cognitive ability test can result in differential predictive validity of academic performance. Personality and Individual Differences, 52(4),497-502.

Chapell,M.,Blanding,B.,Silverstein,M.,Takahashi,M.,Newman,B.,Gubi,A.,y McCann, N. (2005). Test Anxiety and Academic Performance in Undergraduate and Graduate Students. Journal of Educational Psychology, 97(2), 268-274.

Fundación BBVA e Instituto Valenciano de Investigaciones Económicas. (2017). U-Ranking 2017. Indicadores sintéticos de las universidades españolas. Recuperado dehttps://drive.google.com/file/d/0B9DluxBCJhBoN0pBQVFKemd4SGc/view.

García-Ros, R., Pérez-González, F., Pérez-Blasco, J., y Natividad, L.. (2012). Evaluación del estrés académico en estudiantes de nueva incorporación a la universidad. Revista Latinoamericana de Psicología, 44(2),143-154.

Gargallo, B., Pérez, C., Serra, B., Sánchez, F. J., Ros, I. (2007-A). Actitudes ante el aprendizaje y rendimiento académico en estudiantes universitarios. Revista Iberoamericana de Educación, 42(1),1-11.

Gargallo, B., Suárez, J., y Ferreras, A. (2007-B). Estrategias de aprendizaje y rendimiento académico en estudiantes universitarios. Revista de Investigación Educativa, 25(2), 421- 441.

Gargallo, B., Pérez, C., Fernández, A., y Jiménez, M. A. (2007-C-). La evaluación de las actitudes ante el aprendizaje de los estudiantes universitarios. El cuestionario CEVAPU. Revista Electrónica Teoría de la Educación, 8(2),238-256.

Gargallo, B., Suárez-Rodríguez, J. M., y Pérez-Pérez, C. (2009). El cuestionario CEVEAPEU. Un instrumento para la evaluación de las estrategias de aprendizaje de los estudiantes universitarios. Relieve, 15(2),1-31.

González-Peiteado, M., Pino-Juste, M., y Penado-Abilleira, M. (2016). Valoración psicométrica de un cuestionario para medir la satisfacción con la experiencia universitaria. Revista Magis. En prensa.

Hamaideh, S. H., Al-Omari, H., y Al-Modallal, H. (2017). Nursing students' perceived stress and coping behaviors in clinical training in Saudi Arabia. Journal of Mental Health, 27(3), 197-203.

Juárez, C. S., Rodríguez, G., Escoto, M. C. y Luna, E. (2016). Relación de los estilos y estrategias de aprendizaje con el rendimiento académico en estudiantes universitarios. Revista de Estilos de Aprendizaje, 9(17),268-288.

Khalaila R. (2015) The relationship between academic self-concept, intrinsic motivation, test anxiety, and academic achievement among nursing students: Mediating and moderating effects. Nurse Education Today, 35(3),432-438.

Kember, D., y Leung, D. Y. P. (2005). The influence of active learning experiences on the development of graduate capabilities. Studies in Higher Education, 30(2),155-170.

Lang, J. W. B., y Lang, J. (2010). Priming competence diminishes the link between cognitive test anxiety and test performance. Implications for the interpretation of test scores. Psychological Science, 21(6),811-819.

LePine, J. A., LePine, M. A., y Jackson, C. L. (2004). Challenge and hindrance stress: Relationships with exhaustion, motivation to learn, and learning performance. Journal of Applied Psychology, 89(5),883-91.

Palacio, J. E., Caballero, C. C., González, O., Gravini. M., y Contreras, K. P. (2012). Relación del burnout y las estrategias de afrontamiento con el promedio académico en estudiantes universitarios. Universitas Psychologica, 11(2),535-544.

Pulido, M. A., Serrano, M. L., Valdés, E., Chávez, M. T., Hidalgo, P. y Vera, F. (2011). Estrés académico en estudiantes universitarios. Psicología y Salud, 21(1),31-37.

Rausch, J. L., y Hamilton, M. W. (2006). Goals and Distractions: Explanations of Early Attrition from Traditional University Freshmen. The Qualitative Report, 11(2),317-334.

Rayle, A. D., y Chung, K. (2007). Revisiting first-year college students' mattering: Social support, academic stress, and the mattering experience. Journal of College Students Retention: Research, Theory and Practice, 9(1),21-37.

Robotham, D., y Julian, C. (2006). Stress and the higher education student: A critical review of the literature. Journal of Further and Higher Education, 30(2),107-117.

Salanova, M., Martínez, I. M., Breso, E., Llorens, S., y Grau, R. (2005). Bienestar psicológico en estudiantes universitarios: facilitadores y obstaculizadores del desempeño académico. Anales de Psicología, 21(1),170-180.

Torre, J. C. (2007). La autoeficacia, la autorregulación y los enfoques de aprendizaje en estudiantes universitarios (Tesis doctoral). Universidad Pontificia Comillas,Madrid. 
Valero, L. (1999) Evaluación de ansiedad ante exámenes: Datos de aplicación y fiabilidad de un cuestionario CAEX. Anales de Psicología, 15(2),223-231.

Yip, M. C. W. (2012). Learning strategies and self-efficacy as predictors of academic performance: a preliminary study. Quality in Higher Education, 18(1),23-34. 